\title{
Protocol
}

\section{Effectiveness of modified Whitfield's ointment with oral griseofulvin in the treatment of dermatophytosis poorly responsive to standard antifungal therapy: a randomized, double blind, within patient placebo controlled study}

\author{
Sanjeewani Fonseka ${ }^{1}$, Brabaharan Subhani ${ }^{1}$, P. Vitharana Ranjith Kumarasiri ${ }^{2}$
}

Department of Pharmacology, ${ }^{2}$ Department of Community Medicine, University of Peradeniya, Sri Lanka

Received: 30 July 2019

Revised: 12 September 2019

Accepted: 16 September 2019

*Correspondence:

Dr. Sanjeewani Fonseka,

E-mail: sanjeewani.fonseka@yahoo.com

Copyright: () the author(s), publisher and licensee Medip Academy. This is an open-access article distributed under the terms of the Creative Commons Attribution Non-Commercial License, which permits unrestricted non-commercial use. distribution. and reproduction in anv medium. provided the original work is properlv cited.

\section{ABSTRACT}

Background: Dermatophytosis is a superficial fungal infection found in hot and humid areas particularly in tropical regions and affects the keratinized regions of the body. It is usually treated with a combination of topical and systemic antifungal therapy as well as improved hygienic measures. Over the last few decades there has been an increase in the prevalence of dermatophyte infections which are poorly responding to standard antifungal therapy.

Methods: Modified Whitfield's ointment is a combination of 5\%-5\% Salicylic acid and Benzoic acid with an emulsifying ointment as a vehicle which has both a fungistatic and a keratolytic action. Oral Griseofulvin is a systemic antifungal agent which is a fungistatic agent. The combination of the above agents is synergistic. A randomized double blind, within-patient-placebo-controlled trial was designed for the treatment of dermatophytosis poorly responsive to standard antifungal therapy.

Conclusions: This may shed light on the treatment of dermatophytosis poorly responsive to standard antifungal therapy.

Trial Registration: This trial is registered with WHO trial registry number (Universal trial number): U111-12358791.

Keywords: Dermatophytosis, Modified Whitfield's ointment, Griseofulvin, Clinical trial

\section{INTRODUCTION}

Dermatophytosis is a superficial fungal infection which causes of superficial skin infections. They are mould fungi adapted to parasitize keratin layer of the skin through the production of proteases and is limited to the stratum corneum. Dermatophytosis is mostly caused by Trichophyton, Microsporum and Epidermophyton spp. transmitted by human, animal or soil contact and occur in hot and humid climatic conditions.

Localized disease usually responds to topical therapy. ${ }^{2}$ Antiseptic preparations such as Whitfield ointment were used in the past. Systemic therapy is generally used for extensive disease and tinea incognito and examples are griseofulvin, fluconazole, itraconazole and terbinafine. These drugs reach effective cutaneous levels but prior to selection of an appropriate agent a number of considerations are necessary, including culture results (and perhaps minimal inhibitory concentration (MIC)), cure rate, cost, complications, drug interactions, convenience, compliance, and the age, health, and medical history of the patient. ${ }^{3,4}$ The use of topical agents along with systematic therapy have been shown to have an effect enhancing the action of the antifungals. ${ }^{5}$ 
Last few years have seen the emergence of forms of dermatophytosis which are difficult to treat despite of use of multiple topical and systemic therapy. ${ }^{6}$ This has been documented predominantly in India. At least 3-4\% of dermatophytosis are now considered to be chronic, secondary to host factors such as obesity, changes in patterns of clothing and hygiene, environmental factors such as global warming, migration of labour, or pharmacologic factors. ${ }^{6,7}$ Patients with immunocompromised status, diabetes mellitus, atopy, and intake of systemic corticosteroids are also predisposed to chronic dermatophytosis. ${ }^{8}$

Clinical resistance has been defined as the persistence or progression of an infection despite in vitro activity against the organism. Such failures can be attributed to incorrect diagnosis, immunosuppression, suboptimal dose or duration of therapy and hygienic practices among patients are responsible for spread and persistence of the infection despite of treatment. ${ }^{9}$ A successful clinical response depends on the susceptibility of the pathogenic organism, the host immune system, drug penetration and distribution, patient compliance, and absence of a protected or persistent focus of infection. ${ }^{8}$ In vivo resistance is also correlated with antifungal misuse because patients often fail to complete the full course of treatment. Thus, the inadequate use or dosage of drugs contributes to the failure in eliminating the disease agent completely, encouraging growth of the most resistant strains, which may lead to hard-to-treat fungal infections. ${ }^{10}$

Some predisposing factors particularly relevant to India which may also be applicable to Sri Lanka are wide overthe-counter (OTC) availability and rampant use of topical steroid and antifungal combinations by the patients themselves or unrestricted prescription of these products by quacks and general practitioners. ${ }^{11,12}$ The above has led to altered cell mediated immunity in the skin and alteration in the morphological patterns of the infection making diagnosis a challenge. ${ }^{13}$

Clinical Trichophyton rubrum strain has been found to exhibit primary resistance to terbinafine. ${ }^{14}$ An amino acid substitution has been linked to primary resistance observed among patients. ${ }^{15}$

Benzoic acid and Salicylic acid ointment known as modified Whitfield's ointment combines the fungistatic action of benzoate with the keratolytic action of salicylate. The traditional half strength formulation contains Benzoic acid and Salicylic acid in a ratio 2:1 (usually 6\%:3\%). Benzoic acid is a fungistatic agent, hence eradication of the infection occurs only after the infected stratum corneum is shed, and continuous medication is required for weeks to months. Salicylic acid is rapidly absorbed by the skin and causes the keratinocytes to swell, soften and desquamate. It causes mild irritation at the site of application. ${ }^{16}$
Griseofulvin inhibits microtubule function and hence disrupts assembly of the mitotic spindle. It is given via the oral route and peak concentration of the drug is reached within 4 hours with variable blood levels and is detectable in the stratum corneum of the skin in 4-8 hours. Absorption may be enhanced by giving it with a fatty meal. The unwanted side effects of treatment include headache, nausea, vomiting, diarrhoea, dry mouth and hepatotoxicity. Griseofulvin is categorized as a class C (a probable but rare cause of clinically apparent liver injury) drug in terms of liver injury. It may cause mild elevation of transaminases but is usually asymptomatic and resolve even with continuation of medication. The documented incidence of acute severe liver injury is less than $1 \%$ and does not warrant routine assessment of liver enzymes. $^{16,17}$

There are few trials evaluating the standard and modified preparations of the Whitfield ointment which is still a widely used treatment in many parts of the world. Although there is widespread, global availability of many of the interventions, in reality many people, particularly in resource-poor countries, may only have access to older treatments such as Whitfield's ointment or even Castellani's paint.

Benzoic acid is an irritant to the skin and most patients find it difficult to tolerate high percentages of benzoic acid, especially in hot humid climates and hence the adherence to treatment is poor. Low percentages of salicylic acid do not remove the stratum corneum adequately and therefore, drug penetration to deeper layers of the epidermis may not happen adequately. Taking these factors into consideration, World Health Organization (WHO) in its publication Dermatological preparations for the Tropics has suggested a modified Whitfield ointment with $5 \%$ benzoic acid and $5 \%$ salicylic acid for tropical countries. ${ }^{18}$

Hence it is possible to combine the keratolytic effect of Salicylic acid and the fungistatic properties of both benzoic acid and oral griseofulvin and exploit their synergistic action to successfully treat treatment resistant dermatophytosis.

There is a rising incidence of dermatophytosis poorly responsive to standard antifungal therapy documented in Asian countries which are developing nations often relying on underfunded public health systems. Hence there is a need for cost-effective treatment options to curb the challenge of poorly resolving forms of dermatophytosis. The possible approaches are the use of combined systemic antifungals and modified versions of previously used treatments such as Whitfield ointment. Oral griseofulvin is an accepted and time tested systemic fungal used in the treatment of dermatophytosis. The dose required in $2.3 \mathrm{mg} / \mathrm{kg}$ hence a dose of $500-1000 \mathrm{mg}$ for an adult. Due to the mechanism of action griseofulvin needs to be given over a long period for the treatment to be effective. Modified Whitfield's ointment is a 
fungistatic agent composed of salicylic acid, benzoic acid and emulsifying ointment. Salicylic acid has a keratolytic action which enhances the systemic drug penetration and cutaneous drug levels of oral griseofulvin hence enabling effective and persistent levels of the drug. ${ }^{1}$

There is a scarcity of studies on dermatophytosis poorly responsive to standard antifungal therapy which is increasingly becoming a challenge in clinical practice. Since most of these patients have failed to respond to conventional systemic antifungals, we intend to evaluate the effectiveness of the combination of oral griseofulvin and topical modified Whitfield's ointment in dermatophytosis poorly responsive to standard antifungal therapy.

Objectives will be to assess the effectiveness and safety of topical modified Whitfield's ointment applied twice daily along with oral griseofulvin $500 \mathrm{mg}$ daily in adults with dermatophytosis poorly responding to standard antifungal therapy. Thus, we hypothesize that modified
Whitfield's ointment with oral griseofulvin given over a period of 8 weeks is effective in the treatment of poorly resolving forms of dermatophytosis due to the synergistic nature of the two treatments.

\section{METHODS}

\section{Trial design}

The study will be conducted as a randomized double blind, within-patient-placebo-controlled trial. All will be part of both treatment arms. The response as well as the rate of healing is dependent on a myriad of factors ranging from immunogenic factors, hygienic practices and environmental factors. Any patient-related factors that may have determined the outcome of the treatment will be controlled by the within-patient design. This protocol was designed complying with the Consolidated Standards of Reporting Trials (CONSORT) statement. Figure 1 provides an overview of the study as a CONSORT trial flow chart.

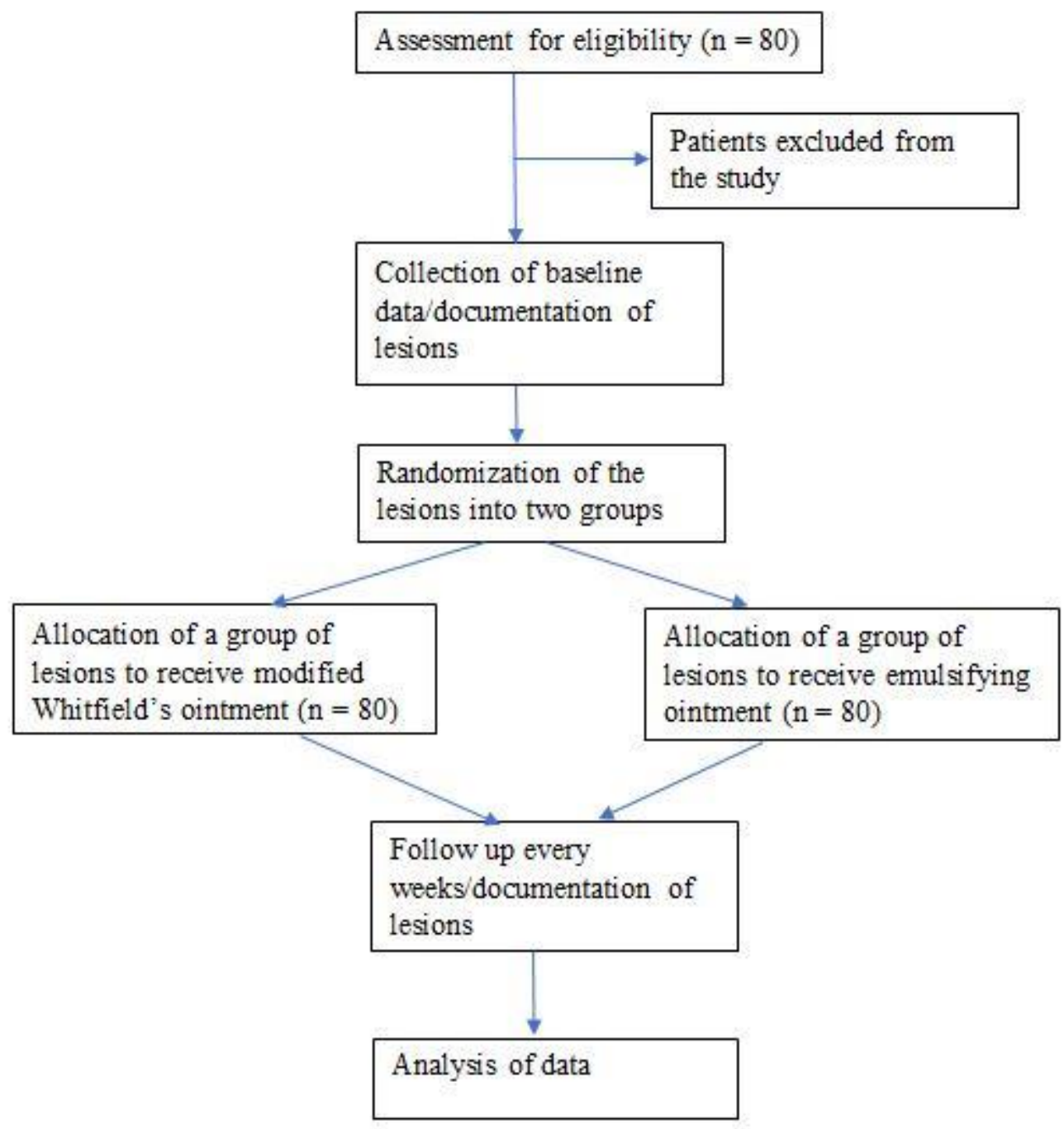

Figure 1: CONSORT flow diagram for the trial. 


\section{Participants}

The participants will be males or females aged more than 18 years who will provide informed written consent, with clinically confirmed dermatophytosis, poorly responsive to standard antifungal therapy (dermatophytosis for a duration of 3 months or more which has been treated with 2 or more systemic antifungals) and with the presence of two or more skin lesions caused by dermatophytosis.

The following categories of patients will be excluded from the study: patients who did not give consent, patients with contraindications for griseofulvin such as chronic liver disease, acute porphyria, recent exacerbation of systemic lupus erythematosus, patients who have a history of adverse reactions to the ingredients of the modified Whitfield's ointment, pregnancy (excluded by the last menstrual period, if unsure of the dates, treatment was commenced after the next menstrual period), a male patients who wanted to father a child within the next 6 months, women who were breast feeding, patients with lesions involving the nails and scalp and patients with mental disorders who are unlikely to adhere to the instructions and treatment procedure of the study.

The patients will be recruited from the outpatient dermatology clinic "Skin Center", Sirimavo Bandaranayake Mawatha, Kandy, Sri Lanka. The study will be conducted at the Department of Pharmacology, Faculty of Medicine University of Peradeniya, Sri Lanka. The patients will be enrolled for duration of 22 months.

\section{Interventions}

All patients will be screened based on the eligibility criteria. Past medical history/medication history will be reviewed. The patients who fulfil the criteria were selected and informed written consent was obtained by a medically qualified co-investigator who was not involved in the routine medical care of the patient. Consent will be sought and documented by explaining the nature of the study, probable risks, benefits and the follow up visits that would be needed to be carried out. Demographic data, prior treatment history for dermatophytosis, comorbidities and concomitant medications will be documented. If the patients will be able to be followed up for duration of 8 weeks and attend regular fortnightly reviews, they were recruited to the study. Patients were informed to continue any medications that they may have taken previously and attend any clinics they may have attended previously. At the initial visit symptoms based on visual analogue score (VAS) and examination findings (the examination was carried out by the principal investigator (PI) who was a consultant dermatologist and findings documented in accordance with the 4-point Lickert scale (absent, mild, moderate, severe)). Lesions will be documented on a diagram of the data collection sheet and traced on to tracing paper and the area of the lesion calculated by the grid method.
Based on the location and the number of lesions, they will be randomized into two groups based on a flip of a coin. Skin scrapings will be taken for direct microscopy from both groups of lesions.

The allocation of the lesions to the two arms will be documented on a paper with a human figure and placed within an envelope which contained the trial number, name and address. The envelopes were placed under lock and key. The envelopes will be opened at each subsequent visit only by the pharmacist and a single coinvestigator before dispensing the treatment as they will be responsible for dispensing the treatment.

Lesions in one group will receive the modified Whitfield's ointment whereas the lesions on the other group received the emulsifying ointment without any active ingredients.

All patients will be dispensed oral griseofulvin $500 \mathrm{mg}$ which is a drug dispensed as a tablet. The griseofulvin will be purchased in bulk and the quantity required for two weeks (14 tablets) duration was dispensed to the patients.

Benzoic acid, salicylic acid and components of the emulsifying base: emulsifying wax, white soft paraffin and liquid paraffin are all elemental compounds. The above compounds will be procured from and the modified Whitfield's ointment will be prepared by a qualified pharmacist at the department of Pharmacology.

The emulsifying ointment will be prepared with $50 \%$ white soft paraffin, $30 \%$ emulsifying wax and $20 \%$ liquid paraffin. White soft paraffin, liquid paraffin and emulsifying wax will be placed within an evaporative dish and melted to together in a water bath at $60^{\circ} \mathrm{C}$. The ointment will be made according to the recommendations of WHO's "Dermatological Preparations of the Tropics" and the formula stated in the electronic medicines' compendium. Benzoic acid and salicylic acid will be added to the prepared emulsifying ointment to make the modified whitfield ointment (benzoic acid-5\%, salicylic acid-5\%) as stated in WHO's "Dermatological Preparations of the Tropics". Fifty grams of the modified Whitfield's ointment and the emulsifying ointment will be placed within standard plastic containers by the pharmacist. The containers were labelled with "instructions for external use only, apply twice daily, keep away from children, store in a cool dry place, with the date of manufacture and expiration". The modified Whitfield's ointment has a shelf life of approximately 3 months. The emulsifying ointment has a shelf life of 2 years.

All patients received oral griseofulvin $500 \mathrm{mg}$ at night and be advised to take the drug with a glass of milk or a fatty meal. The patients needed to apply the ointments twice daily. The patients were provided with two wooden spatulas for the two ointments. They were asked to take 
the required amount of the ointment with a wooden spatula, get it onto the palm of the hand, and apply it on to the lesions by using a fingertip until an adequate layer is present to cover the lesions and to include $1 \mathrm{~cm}$ outside the active edge of the lesion.

The patients will be asked not to re-insert the left-over ointment to the container and wash the spatula before applying ointment the next time. All patients will be given a demonstration on the method of application of treatment and a leaflet on how to apply the medication to ensure uniformity of application of the two medications and the importance of maintaining proper hygiene. A demonstration of how to apply the ointment will be done at each visit.

If the lesion was located at a site that is not reachable to the patient's hand (e.g., lesions on the back) the ointment was applied by another person. A patient diagnosed of having dermatophyte infections on the flexural surfaces will be asked to apply a thin layer of the Whitfield's ointment and the emulsifying ointment on the respective groups of lesions to minimize irritation. However, if the patient found the ointment to be irritating, a $\mathrm{ZnO}$ paste will be dispensed to the patient and requested to apply it over the fold which would act as a barrier. The ointments will applied away from the layer of $\mathrm{ZnO}$. The area of the lesion covered by $\mathrm{ZnO}$ was deducted from the surface area of the lesion during the assessment.

Containers of emulsifying ointment and modified Whitfield's ointment will be stored in a cool dry place in the Department of Pharmacology. The extra chemicals will be stored within the laboratory at the Department of Pharmacology. The drugs were stored in the Department of Pharmacology in a cool dry place and the patients were informed of keeping the drugs in a cool dry place in their homes away from children.

Patients will be instructed to bring all left-over medication to the clinic at each visit. The investigator will be responsible for the assessment of patient compliance by inquiring from the patient as to whether he/she has administered the medication according to the instructions given, counting the number of tablets of griseofulvin left and checking the left-over ointment in the container. The adherence to treatment will be documented on the data collection sheet. Patients will be given a chart to complete each time they apply ointments and take griseofulvin tablets. This chart will be reviewed at each follow up visit. All patients will be encouraged/counselled on the importance of adherence to instructions during each visit.

Each patient will be followed up every 2 weeks for a duration of 8 weeks which will be a total of 4 follow-up visits. At each subsequent visit symptoms based on VAS and examination findings (the examination will be carried out by the PI and findings documented in accordance with the 4-point Lickert scale (absent, mild, moderate, severe) will be carried out. Lesions will be documented in a diagram of the data collection sheet and traced on to tracing paper and the area of the lesion calculated by the grid method. Skin scrapings will be taken for direct microscopy from both groups of lesions. Any adverse events and adherence to treatment will be documented.

The lesions will be documented on a diagram and a copy given to the patients to ensure that the appropriate treatment was carried out on the appropriate group of lesions. Immediately prior to assessment of the lesions the above-mentioned copy will be given to the pharmacist to prevent bias during the assessment of the lesions.

\section{Outcomes}

The primary outcome measures will be clinical assessment of each lesion: symptoms and overall improvement based on VAS, examination findings (The examination will be carried out by the PI and findings documented in accordance with the 4-point Lickert scale) and assessment of each lesion by calculating the surface area of the lesions by the tracing paper and grid method. The secondary outcome measures will be mycological assessment by direct microscopy of skin scrapings of the two groups of lesions (KOH-20\%+Parker's Indian ink) and adverse events. To minimize errors in measurement all co-investigators will be trained in the assessment of the lesion prior to the commencement of study by the PI.

\section{Sample size}

In sample size calculation the alpha value will be taken to be 0.05 , beta value taken to be 0.05 , with an effects size for the existing systemic treatment of poorly resolving dermatophytosis of $0.40 .{ }^{19,20}$ The postulated cure rate with the new treatment was taken to be 0.70 . Hence a sample size of 65 was calculated.

$N=\frac{P 1 \times(100-P 1)+P 2(100-P 2) \times f(\alpha, \beta)}{(P 2-P 1)^{2}}$

where $\mathrm{P} 1=40, \mathrm{P} 2=70, \mathrm{f}(\alpha, \beta)=13$, so $\mathrm{N}=65$.

Considering a dropout rate/withdrawal rate of $20 \%$ the total sample size was calculated to be 78 and rounded to a value of 80 . Data will be analysed at the end of the study. The data from patients who have withdrawn from the study will not be analysed.

\section{Randomization}

\section{Sequence generation}

The body will arbitrarily be divided into two parts as right and left as shown in Figure 2. A coin will be tossed and the lesions will be randomized to the two sides. If it as "Heads" the modified Whitfield's ointment will be applied to the right side of the body whereas the 
emulsifying ointment will be applied to the left side of the body. If it is "Tails" the emulsifying ointment was applied to the right side of the body whereas the modified Whitfield's Ointment was applied to the left side of the body.

\section{Right (1)}

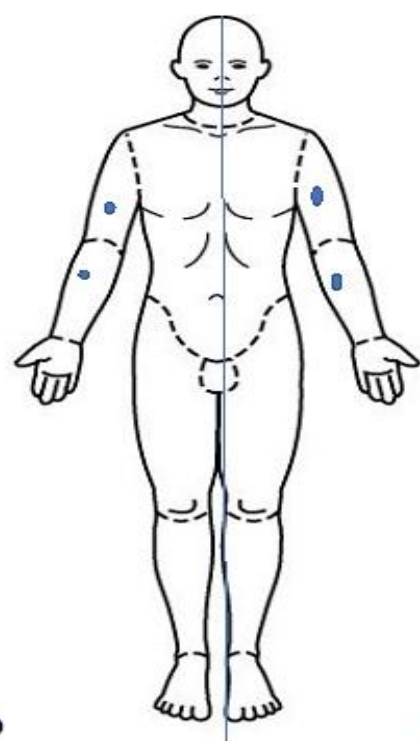

Left (2)

Figure 2: Randomization of lesions if the lesions are situated bilaterally.

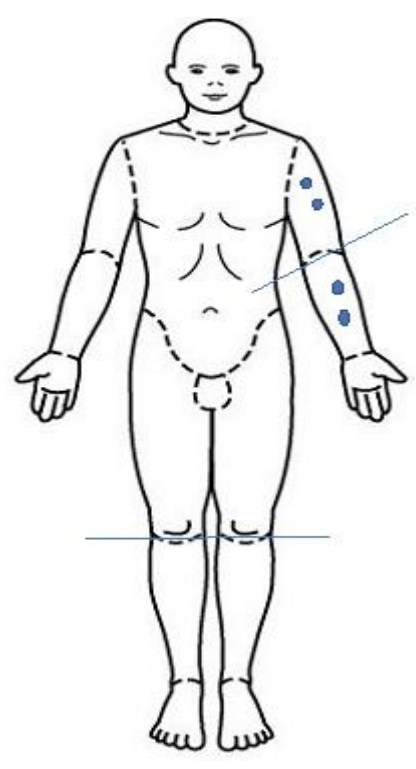

Proximal (1)

Distal (2)

Figure 3: Randomization of lesions if the lesions are confined to a single extremity.

If the lesions are confined to a single extremity it was divided into two parts as proximal and distal as shown in Figure 3. A coin was tossed. If it is "Heads" the modified Whitfield's ointment was applied to the lesions on the proximal part whereas the emulsifying ointment was applied to the distal part of the body. If it is "Tails" the emulsifying ointment was applied to the lesions on the proximal part whereas the modified Whitfield's ointment was applied to the lesions on the distal part of the body.
If the lesions were located unilaterally in the upper and lower limbs and torso, the body was divided into upper and lower two parts by a line passing through the umbilicus as shown in Figure 4. The lesions were divided as upper and lower and a coin was tossed. If it is "Heads", the modified Whitfield's ointment was applied to the upper half of the body whereas the emulsifying ointment was applied to the lower half of the body. If it is "Tails" the emulsifying ointment was applied to the upper half of the body whereas the modified Whitfield's ointment was applied to the lower half of the body.

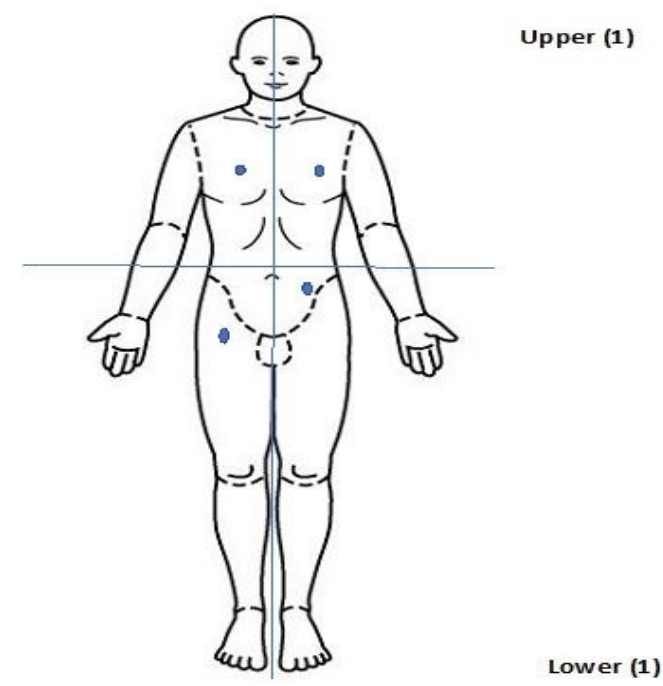

Figure 4: Randomization of lesions if the lesions are located unilaterally.

\section{Allocation concealment mechanism}

Fifty grams each of modified Whitfield's ointment and emulsifying ointment was placed within identical containers by the pharmacist. The ointments were prepared in batches and each ointment would contain a batch number. A random letter sequence generator was used to generate 3 pairs of letters at the beginning of the manufacturing of the ointments. These letters were assigned to the ointments. The above code was unknown to the Clinician and the assessor. The random Letter Sequence Generator will be used to generate a sequence of letters for example as "G - M" (Combination 1), "J O" (Combination 2) and "Y - N" (Combination 3). The ointments were prepared in batches and each ointment contained a batch number. Each batch was comprised of $1 \mathrm{~kg}$ of modified Whitfield's ointment and $1 \mathrm{~kg}$ of emulsifying ointment and will be prepared every two weeks approximately. The modified Whitfield's ointment (the first letter of the combination) was placed in containers marked "G", "J" and "Y". The emulsifying ointment (the second letter of the combination) was placed in containers marked "M", "O" and "N". A random number generator will be used to generate a sequence of numbers containing 100 numbers and 3 columns for the 3 combinations of treatments. The order 
in which the treatment was dispensed was based on the above sequence that was generated.

\section{Implementation}

The random sequence will be generated by the trial coordinator. The allocation of the lesions to the two arms will be documented on a paper with a human figure and placed within an envelope which contained the trial number, name and address. The envelopes were placed under lock and key. The envelopes were opened at each subsequent visit only by the pharmacist before dispensing the treatment. A record of the above allocation along with the batch number was maintained by the trial pharmacist and a co-investigator who was to act as the trial coordinator were aware of the allocation in each patient.

\section{Blinding}

The clinician as well as the assessor will be blind to the allocation of the groups of lesions.

\section{Statistical methods}

The primary end point of the study will be complete clinical clearance of the lesions. The area of the lesions will be measured during each visit. A factorial study design will be carried out with a $2 \times 2$ ANOVA repeated measures. Mycological clearance will be documented and assessed by a chi-square test. A descriptive analysis of the clinical features and adverse events encountered by the patients will be carried out.

\section{DISCUSSION}

This article represents a protocol of a study to assess the treatment of resistant forms of dermatophytosis which will be conducted as a randomized double blind, withinpatient-placebo-controlled trial. Chronic dermatophytosis is a common condition in Asia contributed for by the climatic conditions, hygienic practices and the treatment patterns.

Griseofulvin (1000 mg/day) compared with ketoconazole (200 mg/day), both for 4 weeks of treatment, and no difference in cure rates was seen. The trial had a total of 29 participants and hence inadequately powered. ${ }^{21}$ Two small trials compared griseofulvin (500 mg/day) with terbinafine $(250 \mathrm{mg} /$ day $)$ for either 4 or 6 weeks. Both trials showed that terbinafine had statistically significantly better cure rates, $100 \%$ and $86 \%$, whereas griseofulvin achieved $50 \%$ and $33 \% .^{22}$ A comparison of oral ketoconazole and griseofulvin revealed an almost equal percentage, $75 \%$ and $74 \%$ respectively of improvement though there was a species-based variation of the response to treatment. ${ }^{23}$

Antifungal susceptibility testing showed high MIC values obtained for Trichophyton spp. and $50 \%$ of the other species for terbinafine and griseofulvin. ${ }^{24}$ Brazilian studies have revealed resistance of many strains to griseofulvin but in vitro antifungal susceptibility of dermatophytosis for the griseofulvin and terbinafine combination. $^{24,25}$

Within patient studies though largely uncommon has been documented in the field of Dermatology due to the superficial nature of the lesions as well as the use of topical treatment which are often unlikely to have a systemic effect. A randomized, double-blind, activecontrolled within patient design was used to compare the effectiveness of Fenticonazole (2\%) cream b.i.d. for up to maximum of 4 weeks versus clotrimazole $(1 \%)$ cream b.i.d. for up to a maximum of 4 weeks. Patients with bilateral symmetrical lesions were included in the study and assessed with both clinical and mycological outcome measures. Further assessment of the number of the lesions was carried out and treatment given and the percentage cure rate for the lesions was calculated. ${ }^{26,27}$

The small randomized control trial (RCT) trial of Whitfield's ointment and Variotin applied for eight weeks only included ten people with athlete's foot and it is therefore unsurprising that the trialists failed to detect differences between these two compounds. ${ }^{28}$ Combining salicylic acid with nitrite produces greater effectiveness than that of salicylic acid alone. ${ }^{29}$

Whitfield's ointment versus clotrimazole (1\%) cream each applied three times a day. Based on a negative culture after two weeks, the standard Whitfield's ointment was less effective than clotrimazole $(6 / 28$ compared to $16 / 27 \mathrm{p}=0.01)$. The standard Whitfield's ointment was less effective than clotrimazole after two weeks with 14/28 of participants judged as marked improvement' to 'healed' compared with 21/27 participants $(\mathrm{p}=0.04)$. No adverse effects were reported. ${ }^{30}$ Based on a negative culture after two weeks, Whitfield's ointment was less effective than tolnaftate in achieving mycological cure, with $6 / 28$ cured compared to $12 / 19$ $(\mathrm{p}=0.007){ }^{31}$

The combination of oral griseofulvin which is a time tested and cost effective drug for the treatment of dermatophytosis along with a modified form of Whitfield's ointment which is a keratolytic along with the uniformity in the administration of treatment and advices on appropriate hygienic practices may be a suitable mode of therapy for the treatment of patients with dermatophytosis poorly responsive to standard therapy.

\section{CONCLUSIONS}

This may shed light on the treatment of dermatophytosis poorly responsive to standard antifungal therapy.

Funding: University Research Grant, Faculty of Medicine, University of Peradeniya, Sri Lanka

Conflict of interest: None declared

Ethical approval: The study was approved by the Institutional Ethics Committee 


\section{REFERENCES}

1. White JFPHTJGKDLN. Manson's Tropical Diseases. 23rd edition. Saunder's Ltd; 2013: 1360.

2. Gooskens V, Pönnighaus JM, Clayton Y, Mkandawire P, Sterne JAC. Treatment of superficial mycoses in the tropics: Whitfield's ointment versus clotrimazole. Int Dermatol. 1994;33(10):738-42.

3. Gupta AK, Ryder JE, Chow M, Cooper EA. Dermatophytosis: the management of fungal infections. Skinmed. 2005;4(5):305-10.

4. Lesher JL. Oral therapy of common superficial fungal infections of the skin. Am Acad Dermatol. 1999;40(6):S31-4.

5. Tamura T, Asahara M, Yamamoto M, Yamaura M, Matsumura M, Goto K, et al. In vitro susceptibility of dermatomycoses agents to six antifungal drugs and evaluation by fractional inhibitory concentration index of combined effects of amorolfine and itraconazole in dermatophytes. Microbiol Immunol. 2014;58(1):1-8.

6. Rengasamy M, Chellam J, Ganapati S. Systemic therapy of dermatophytosis: Practical and systematic approach. Clin Dermatol Rev. 2017;1(3):19-23.

7. Sharma M, Palanichamy T. Psychosocial interventions for technological addictions. Indian $\mathbf{J}$ Pathol Microbiol. 2017;60(4):541-5.

8. Dogra S, Uprety S. The menace of chronic and recurrent dermatophytosis in India: Is the problem deeper than we perceive? Indian Dermatol Online J. 2016;7(2):73-6.

9. Singh S, Verma P, Chandra U, Tiwary N. Risk factors for chronic and chronic-relapsing tinea corporis, tinea cruris and tinea faciei: Results of a case\&\#8211; control study. Indian J Dermatol Venereol Leprol. 2019;85(2):197-200.

10. Panda S, Verma S. The menace of dermatophytosis in India: The evidence that we need. Indian $\mathbf{J}$ Dermatol Venereol Leprol. 2017;83(3):281-4.

11. Verma SB. Emergence of recalcitrant dermatophytosis in India. Lancet Infect Dis. 2018;18(7):718-9.

12. Sonthalia $S$, Agrawal $M$, Goldust $M$, Das $S$, Bhattacharya SN. Antifungal therapeutic failures in India: an important issue being overlooked. Lancet Infect Dis. 2018;18(11):1181-2.

13. Bishnoi A, Vinay K, Dogra S. Emergence of recalcitrant dermatophytosis in India. Lancet Infect Dis. 2018;18(3):250-1.

14. Mukherjee PK, Leidich SD, Isham N, Leitner I, Ryder NS, Ghannoum MA. Clinical Trichophyton rubrum strain exhibiting primary resistance to terbinafine. Antimicrob Agents Chemotherapy. 2003;47(1):82-6.

15. Osborne CS, Leitner I, Favre B, Ryder NS. Amino acid substitution in Trichophyton rubrum squalene epoxidase associated with resistance to terbinafine. Antimicrob Agents Chemotherapy. 2005;49(7):2840-4.
16. Goodman and Gilman's The Pharmacological Basis of Therapies. 12 edition. McGraw-Hill; 2011.

17. NIH. Livertox: NIH: U.S. National Library of Medicine; 2019. Drug Record: Griseofulvin]. Available from: https://livertox.nih.gov/Griseofulvin.htm. Accessed 17 July 2019.

18. Peter Bakker HW, Vincent Gooskens, Ben Naafs, Rachel van der Kaaij, Nicolien Wieringa. Dermatological Preparations for the Tropics. 2nd edition. Beta Science Shop, University of Gronigren, Netherlands; 2012.

19. Bell-Syer SEM, Khan SM, Torgerson DJ. Oral treatments for fungal infections of the skin of the foot. Cochrane Database of Systematic Reviews. 2012;(10).

20. Crawford F, Hollis S. Topical treatments for fungal infections of the skin and nails of the foot. The Cochrane database of systematic reviews. 2007;(3):Cd001434.

21. Roberts DT, Cox NH, Gentles JC, Babu KK. Comparison of ketoconazole and griseofulvin in the treatment of tinea pedis. J Med Veterinary Mycol. 1987;25(5):347-50.

22. Savin RC. Oral terbinafine versus griseofulvin in the treatment of moccasin-type tinea pedis. J Am Acad Dermatol. 1990;23(4 Pt 2):807-9.

23. Hay RJ, Clayton YM, Griffiths WA, Dowd PM. A comparative double blind study of ketoconazole and griseofulvin in dermatophytosis. British J Dermatol. 1985;112(6):691-6.

24. Dabas Y, Xess I, Singh G, Pandey M, Meena S. Molecular Identification and Antifungal Susceptibility Patterns of Clinical Dermatophytes Following CLSI and EUCAST Guidelines. J Fungi. 2017;3(2):17.

25. Lana AJD, Pippi B, Carvalho AR, Moraes RC, Kaiser S, Ortega GG, et al. In Vitro additive effect on griseofulvin and terbinafine combinations against multidrug-resistant dermatophytes. Brazilian J Pharmaceutical Sci. 2018;54.

26. Galuppi R, Gambarara A, Bonoli C, Ostanello F, Tampieri M. Antimycotic effectiveness against dermatophytes: Comparison of two in vitro tests. 2010;:S57-61.

27. Finzi A, Fioroni A, Preti PM, Mounari M. A Double Blind Evaluation of Fenticonazole Cream 2\% and Clotrimazole Cream $1 \%$ in Dermatomycoses/Doppelblindstudie mit Fenticonazol-Creme 2\%ig und Clotrimazol-Creme $1 \%$ bei Dermatomykosen. Mycoses. 1986;29(1):41-4.

28. Zaun H, Luszpinski P. Multicenter double-blind contralateral comparison of naftifin and clotrimazole cream in patients with dermatophytosis and candidiasis. Zeitschrift fur Hautkrankheiten. 1984;59(18):1209-17.

29. Holti G. A double-blind controlled trial of Whitfield's ointment and variotin in ringworm 
infections with a two year 'follow-up'. Acta Dermato-Venereologica. 1970;50(3):229-31.

30. Weller R, Ormerod AD, Hobson RP, Benjamin NJ. A randomized trial of acidified nitrite cream in the treatment of tinea pedis. J Am Acad Dermatol. 1998;38(4):559-63.

31. Sivayathorn A, Piamphongsant T. Topical Antimycotic Agents for the Treatment of Superficial Dermatophytoses in Thailand: A Double-Blind Study. Mycoses. 1979;22(1):21-4.
Cite this article as: Fonseka S, Subhani B, Kumarasiri PVR. Effectiveness of modified Whitfield's ointment with oral griseofulvin in the treatment of dermatophytosis poorly responsive to standard antifungal therapy: a randomized, double blind, within patient placebo controlled study. Int J Clin Trials 2019;6(4):206-14. 